\title{
ON EFFECTIVE AND OPTICAL RESOLUTIONS OF DIFFRACTION DATA SETS
}

by Ludmila Urzhumtseva ${ }^{3}$,Bruno P. Klaholz ${ }^{1}$ and Alexandre Urzhumtsev ${ }^{1,2^{*}}$

1 IGBMC (Institute of Genetics and of Molecular and Cellular Biology), Department of Integrative Structural Biology, Centre National de la Recherche Scientifique (CNRS) UMR 7104 / Institut National de la Santé de la Recherche Médicale (INSERM) U964 / Université de Strasbourg, 1 rue Laurent Fries, 67404 Illkirch, France.

${ }^{2}$ Université de Lorraine, Physical Department, Vandoeuvre-lès-Nancy, 54506, France.

${ }^{3}$ Architecture et Réactivité de l'ARN, UPR 9002 CNRS, IBMC (Institute of Molecular and Cellular Biology), 15 rue R. Descartes, 67084 Strasbourg, France; Université Louis Pasteur de Strasbourg, Strasbourg, F-67000 France.

\section{Supplementary Material}

\section{Reminder A: the three-dimensional interference function}

In order to calculate the integral

$$
\rho(r)=\rho(|\mathbf{r}|)=\int_{|\mathbf{s}| \leq D} e^{-2 \pi i(\mathbf{r s})} d \mathbf{s}
$$

one may use the spherical symmetry of the resulted function and calculate its value along the axis $\mathbf{O Z}$. In the polar coordinates,

$$
(\mathbf{r s})=z s \cos \theta
$$

where $\theta$ is the angle between $\mathbf{O Z}$ and the vector $\mathbf{s}$ of the length $s$. Therefore, 


$$
\begin{aligned}
& \rho(z)=\int_{0}^{D} \int_{0}^{\pi} \int_{0}^{2 \pi} s^{2} \sin \theta e^{-2 \pi i z \cos \theta} d \varphi d \theta d s=2 \pi \int_{0}^{D} \int_{0}^{\pi} s^{2} \sin \theta e^{-2 \pi i z s \cos \theta} d \theta d s= \\
& =-2 \pi \int_{0}^{D} \int_{0}^{\pi} s^{2} e^{-2 \pi i z \cos \theta} d(\cos \theta) d s=2 \pi \int_{0}^{D} \int_{-1}^{1} s^{2} e^{-2 \pi i z s t} d t d s=2 \pi \int_{0}^{D} s^{2} \frac{1}{2 \pi i z s}\left(e^{2 \pi i z s}-e^{-2 \pi i z s}\right) d s= \\
& =\frac{2}{z} \int_{0}^{D} s \sin (2 \pi z s) d s=\frac{2}{z}\left[-\left.\frac{s \cos (2 \pi z s)}{2 \pi z}\right|_{0} ^{D}+\frac{1}{2 \pi z} \int_{0}^{D} \cos (2 \pi z s) d s\right]= \\
& =\frac{1}{\pi z^{2}}\left[-D \cos (2 \pi z D)+\frac{1}{2 \pi z} \sin 2 \pi z s \mid \begin{array}{l}
D \\
0
\end{array}\right]=\frac{1}{2 \pi^{2} z^{3}}[-2 \pi z D \cos (2 \pi z D)+\sin 2 \pi z D]= \\
& =\frac{4 \pi D^{3}}{3}\left[3 \frac{\sin 2 \pi z D-2 \pi z D \cos (2 \pi z D)}{(2 \pi z D)^{3}}\right]
\end{aligned}
$$

With $d=D^{-1}$ and the interference function (Fig. 1a of the main text)

$$
G_{3}(t)=3 \frac{\sin (t)-t \cos (t)}{t^{3}}
$$

we obtain

$$
\rho(r)=\frac{4 \pi d^{-3}}{3} G_{3}(2 \pi r / d)
$$

Obviously, derivatives of this function can be calculated analytically as well. 


\section{Reminder B: some features of the interference function}

Various characteristics of the interference function $G_{3}(t)=G_{3}\left(2 \pi r d^{-1}\right)$ are known.

a) Its first zero is $t \approx 4.49$ corresponding to the distance $r / d \approx 0.715$, and the first minimum is at $r / d \approx 0.917$. In crystallographic literature this was indicated, for example by James (1948) and Stenkamp \& Jensen (1984).

b) Its development in the Taylor series in the origin

$$
\begin{aligned}
& G_{3}(t) \approx \frac{3}{t^{3}}\left[\left(t-\frac{t^{3}}{3 !}+\frac{t^{5}}{5 !}-\frac{t^{7}}{7 !}+\right)-t\left(1-\frac{t^{2}}{2 !}+\frac{t^{4}}{4 !}-\frac{t^{6}}{6 !}\right)\right]= \\
& =\frac{3}{t^{3}}\left[-\frac{t^{3}}{3 !}+\frac{t^{5}}{5 !}-\frac{t^{7}}{7 !}+\frac{t^{3}}{2 !}-\frac{t^{5}}{4 !}+\frac{t^{7}}{6 !}\right]= \\
& =3\left[-\frac{1}{3 !}+\frac{t^{2}}{5 !}-\frac{t^{4}}{7 !}+\frac{1}{2 !}-\frac{t^{2}}{4 !}+\frac{t^{4}}{6 !}\right]=1-\frac{t^{2}}{10}+\frac{t^{4}}{280}
\end{aligned}
$$

proves that $G_{3}(0)=1$ and gives also an approximation

$$
G_{3}(t) \approx \exp \left(-\frac{t^{2}}{2 \sigma_{t}^{2}}\right), \text { with } \sigma_{t}=\sqrt{5}
$$

or

$$
\rho(x) \approx \exp \left(-\frac{(2 \pi x)^{2}}{2 \sigma_{\tau}^{2}}\right)=\exp \left(-\frac{x^{2}}{2 \sigma_{x}^{2}}\right),
$$

with

$$
\sigma_{x}=\frac{\sqrt{5}}{2 \pi} \approx 0.356
$$

used by Vaguine et al. (1999). 
c) The inflection point of the Taylor expansion (B1) is defined by its second derivative

$$
-\frac{2}{10}+\frac{12 t^{2}}{280}=0
$$

giving

$$
t=\sqrt{\frac{70}{15}} \approx 2.160
$$

Converting this to $x$ gives

$$
x=\frac{t}{2 \pi} \approx 0.344
$$

However, a direct calculation of the inflection point as a root of

$$
\begin{aligned}
G_{3}^{\prime \prime}(t) & =\frac{d}{d t}\left\{3\left[\frac{\cos (t)}{t^{3}}-\frac{3 \sin (t)}{t^{4}}+\frac{2 \cos (t)}{t^{3}}+\frac{\sin (t)}{t^{2}}\right]\right\}= \\
& =3 \frac{d}{d t}\left[-\frac{3 \sin (t)}{t^{4}}+\frac{3 \cos (t)}{t^{3}}+\frac{\sin (t)}{t^{2}}\right]= \\
& =3\left[\frac{12 \sin (t)}{t^{5}}-\frac{3 \cos (t)}{t^{4}}-\frac{9 \cos (t)}{t^{4}}-\frac{3 \sin (t)}{t^{3}}-\frac{2 \sin (t)}{t^{3}}+\frac{\cos (t)}{t^{2}}\right]= \\
& =\frac{3}{t^{5}}\left[12 \sin (t)-12 t \cos (t)-5 t^{2} \sin (t)+t^{3} \cos (t)\right]
\end{aligned}
$$

gives $t \approx 2.507$ and $x \approx 0.399$ different from (B6) and (B7), respectively, as well as from (B4).

d) One may note also that for the inflection point

$$
G_{3}(2.507) \approx 0.4991 \approx 0.5=0.5 G_{3}(0)
$$




\section{Reminder C: separation of two equal Gaussian peaks}

It is known that a Gaussian peak

$$
g(r)=e^{-\frac{r^{2}}{2 \sigma^{2}}}
$$

has its inflection point at the distance $\sigma$ because

$$
\frac{d^{2} g(r)}{d r^{2}}=\frac{d}{d r}\left(-\frac{r}{\sigma^{2}} e^{-\frac{r^{2}}{2 \sigma^{2}}}\right)=\frac{d}{d r}\left(-\frac{1}{\sigma^{2}}+\frac{r^{2}}{\sigma^{4}}\right) e^{-\frac{r^{2}}{2 \sigma^{2}}}=0
$$

for $r=\sigma$. This means that two Gaussian peaks defined as

$$
\rho_{G, 0}(r)=\left(\frac{4 \pi}{B}\right)^{3 / 2} e^{-\frac{1}{B}(2 \pi r)^{2}}
$$

are seen separately when the distance between them is larger than the limit value $2 \sigma$, i.e.

$$
d_{o p t}=\sqrt{\frac{B}{2 \pi^{2}}}
$$

